\title{
Multireference coupled electron-pair approximations to the multireference coupled cluster method. The MR-CEPA1 method*
}

\author{
P. J. A. RUTTINK†‡, J. H. vAN LENTHE $\$$ and P. TODOROV \$\$ \\ \$Theoretical Chemistry Group and \\ $\S$ Department of Physical Organic Chemistry, Debye Institute, \\ Utrecht University, Padualaan 143584 CH Utrecht, The Netherlands
}

(Received 19 February 2004; accepted 17 August 2004)

\begin{abstract}
An extension of the CEPA1 method to the multi-reference case is presented, the MR-CEPA1 approach. The method takes the variationally included terms into account as in MRDCEPA and corrects for the exclusion principle violating terms as in closed shell CEPA1. It is shown that this method yields potential energy curves that are close to those of the multi-reference coupled cluster method and parallel the full CI results quite well. The size consistency of the method is as good as the MRDCEPA method and much better than approaches that ignore the VI terms like MR-ACPF and MR-AQCC. A simpler method, where the EPV terms are not corrected on an individual basis for the doubly occupied orbitals, dubbed the multi-reference averaged CEPA1 method (MR-ACEPA), which is akin to methods previously suggested by Szalay et al., is comparable in performance.
\end{abstract}

\section{Introduction}

Restricted CI methods are among the most common and easily applied approaches to introduce electron correlation. Efficient algorithms and computer programs have been developed for its application [1-5]. Since the restricted CI method is not size extensive, much effort has been put into modifying this method in order to get approximate size extensivity. The guidelines used for this purpose are taken from coupled cluster (CC) methods [6, 7], which by using the exponential ansatz are specifically designed to meet this requirement.

For the single reference case, assuming that the HF wave function is dominant in the correlated wave function, this has resulted in the coupled electron pairs approaches (CEPA) [8]. They yield good approximations to the $\mathrm{CC}$ results, which are size-extensive while being much less expensive.

For the multi-reference case, the generalization of the CC method is not straightforward. Indeed, many formalisms are possible in principle, but most of these seem only to be applicable in special cases [9-11]. However, a recent proposal for a state-selective MR-CC

†Corresponding author. Email: p.j.a.ruttink@chem.uu.nl *Dedicated to V. R. Saunders on the occasion of his official retirement. approach [12] seems to be promising in the sense that it is generally applicable and that the results compare favourably with full CI results. Several attempts to generalize the CEPA method to the multi-reference case also exist, such as the MR-ACPF [13], MR-AQCC [14] and MRDCEPA[15] methods. In all these cases a complete active space (CAS) reference set is used.

In order to compare these methods, we start from the linearized version of the MR-CC method (MRCEPA0) [16] in a MRCI-type implementation. This method suffers from several disadvantages leading to numerical results which are no better than those obtained with MRCI calculations. The MR-ACPF and MR-AQCC methods improve on this method by compensating for the exclusion principle violating (EPV) terms [17-19], which are present in the MRCEPA0 formalism. In the MRDCEPA method, on the other hand, no EPV corrections appear. Instead, it is focussed on the redundancies that are automatically introduced into a straightforward generalization of the CEPA method to the multi-reference case, corresponding to the variationally interacting (VI) terms [15]. In the method proposed in this paper we combine the previous approaches into a method, which is capable of approximating MR-CC results closely while retaining the simplicity of the modified CI approach. It is in fact an extension of the MR-AQCC-mc approach proposed by 
Szalay and co-workers [20], the main difference being that the EPV corrections for the inactive orbital space are treated in analogy to the CEPA1 method. Therefore we will use the acronym MR-CEPA1 for our method. In a simpler approach, which is very much like the work of Szalay et al., we will not distinguish between individual inactive orbitals, but use an average shift, resulting in a MR Averaged CEPA (MR-ACEPA) method. Calculations for test systems used for validating the MR-CC method show that for these test cases our methods performs better than any of MR-ACPF, MR-AQCC or MRDCEPA.

We have also tested the performance of several methods with respect to the size consistency condition: $\mathrm{E}(\mathrm{A}+\mathrm{B})=\mathrm{E}(\mathrm{A})+\mathrm{E}(\mathrm{B})$ if $\mathrm{A}$ and $\mathrm{B}$ do not interact. It will be shown that the MR-ACPF and MR-AQCC methods may suffer from large discrepancies for systems with near-degeneracies, e.g. $\mathrm{A}=\mathrm{B}=\mathrm{O}_{2}\left({ }^{3} \sum_{g}^{-}\right)$. The MRDCEPA, MR-CEPA1 and MR-ACEPA methods appear to behave much better in this respect. As will be shown in a following paper [21], this is due to the inclusion of the VI terms. However, our research also suggests that it is impossible to obtain exact size consistency if only diagonal shifts are allowed, as is the case for all currently known implementations of multi-reference generalizations of the CEPA method.

\section{Theory}

In all MRCEPA versions the orbital space is divided into three subspaces, which are determined by the occupations in the reference configurations [15]. The inactive orbitals are doubly occupied in all reference configurations, while the active orbitals have variable occupations and the virtual orbitals are empty. The full CI space is also divided into three subspaces: the $\mathrm{P}$ space includes the reference functions, the $\mathrm{Q}$ space is the interacting space of the $\mathrm{P}$ space and the $\mathrm{R}$ space includes all higher excitations. As in the CC method it is sufficient to consider the $\mathrm{R}$ space as consisting only of the interacting space of the $\mathrm{Q}$ space. In the following the spin-restricted formalism and CAS reference spaces will be used throughout.

The normalized MR-CEPA function has the following form

$$
\Psi_{C}=\sum_{I} c_{I} \Phi_{I}^{P}+\sum_{J} c_{J} \Phi_{J}^{Q}
$$

with

$$
c_{I}=\left\langle\Phi_{I}^{P} \mid \Psi_{C}\right\rangle \quad \text { and } \quad c_{J}=\left\langle\Phi_{J}^{Q} \mid \Psi_{C}\right\rangle
$$

A further division of the excitations will be made according to the excitation class [15]. The excitation class of a function is defined by the number of holes $k$ in the inactive orbital space $(0 \leqslant k \leqslant 2)$ and by the number of particles $\ell$ in the virtual orbital space $(0 \leqslant \ell \leqslant 2)$. All reference functions $\Phi_{I}^{P}$ thus belong to class $(0,0)$. A Q-space function belonging to class $(k, \ell)$ is denoted by $\Phi_{J}^{Q}(k, \ell)$. We also introduce excitation operators, which generate the Q-space functions from the reference functions $\Phi_{I}^{P}$ :

$$
\left|\Phi_{j}^{Q}(k, \ell)\right\rangle=\boldsymbol{E}_{i j}(k, \ell)\left|\Phi_{i}^{P}\right\rangle .
$$

Following the CEPA philosophy, the unlinked contributions of the R-space functions are used to restore the size extensivity. The corresponding corrections to the MRCI equations are implemented by applying diagonal shifts to the H-matrix. This corresponds to using equations of the form:

$$
\begin{gathered}
\left\langle\Phi_{I}^{P}|\boldsymbol{H}-\boldsymbol{E}| \Psi_{C}\right\rangle=0 \\
\left\langle\Phi_{J}^{Q}|\boldsymbol{H}-\boldsymbol{E}| \Psi_{C}\right\rangle+K_{J} c_{J}=0
\end{gathered}
$$

where $K_{J}$ is the diagonal shift for $\Phi_{J}^{Q}$. In the single reference case the shift represents the effect of the triple and higher excitations. The explicit form of $K_{J}$ may be derived from the coupled cluster equations, assuming that products of the single and/or double excitation coefficients give the coefficients of the higher excitations. The normal CI equations are obtained by setting all $K_{J}$ s to 0.

Since equations (3) and (4) are homogeneous, the normalization is arbitrary. For our purpose it is convenient to use intermediate normalization, using the projection of the MR-CEPA function $\Psi_{C}$ to the reference space as the reference function $\Psi_{0}$ :

$$
\begin{gathered}
\Psi_{0}=\sum_{I}\left|\Phi_{I}^{P}\right\rangle\left\langle\Phi_{I}^{P} \mid \Psi_{C}\right\rangle=\sum_{I} c_{I}\left|\Phi_{I}^{P}\right\rangle \\
\text { with } \quad \sum_{I} c_{I}^{2}=1 .
\end{gathered}
$$

The reference energy $\boldsymbol{E}_{0}$ is then obtained as:

$$
\boldsymbol{E}_{0}=\left\langle\Psi_{0}|\boldsymbol{H}| \Psi_{0}\right\rangle=\sum_{I J} c_{I} c_{J}\left\langle\Phi_{I}^{P}|\boldsymbol{H}| \Phi_{J}^{P}\right\rangle
$$

and the correlation energy follows from equation (3):

$$
\boldsymbol{E}_{C}=\boldsymbol{E}-\boldsymbol{E}_{0}=\sum_{J} c_{J}\left\langle\Psi_{0}|\boldsymbol{H}| \Phi_{J}^{Q}\right\rangle
$$


The correlation energy is divided into particle-hole class contributions according to:

$$
\boldsymbol{E}_{C}=\sum_{k=0}^{2} \sum_{\ell=0}^{2} \varepsilon(k, \ell)
$$

with

$$
\varepsilon(k, \ell)=\sum_{J} c_{J}\left\langle\Psi_{0}|\boldsymbol{H}| \Phi_{J}^{Q}(k, \ell)\right\rangle
$$

Note that $\varepsilon(0,0)=0.0$, since we assume a CAS reference space.

The MRCEPA function may also be written as [15]:

$$
\left|\Psi_{C}\right\rangle=\left[1+\boldsymbol{T}+\boldsymbol{P}_{a}\left(\frac{1}{2} \boldsymbol{T}^{2}+\frac{1}{6} \boldsymbol{T}^{3}+\cdots\right)\right]|0\rangle
$$

where $\boldsymbol{T}$ generates all single and double excitations with the appropriate coefficients, and

$$
\boldsymbol{T}=\sum_{\substack{k, \ell=0 \\ \text { except } k=\ell=0}}^{2} \boldsymbol{T}(k, \ell)
$$

and

$$
\boldsymbol{P}_{\boldsymbol{a}}=\sum_{\substack{k, \ell=0 \\ \text { except } k=\ell=0}}^{2} \boldsymbol{P}(k, \ell)
$$

$\boldsymbol{P}(k, \ell)$ projects to the excitations of class $(k, \ell)$.

The effect of the projection by $\boldsymbol{P}_{\boldsymbol{a}}$ may be represented by introducing the topological matrices $\boldsymbol{T}^{k \ell}$, which are defined by

if $(k+p \leq 2 \quad$ and $\quad \ell+q \leq 2) \boldsymbol{T}_{p q}^{k \ell}=1 ; \quad$ else $\quad \boldsymbol{T}_{p q}^{k \ell}=0$

where $k$ and $p$ denote the numbers of holes and $\ell$ and $q$ denote the numbers of particles.

The MR-CEPA function (equation (10)) can now be written

$$
\begin{aligned}
\left|\Psi_{C}\right\rangle & =\left[1+\boldsymbol{T}+\sum_{k \ell} \sum_{p q} \mathbf{T}_{p q}^{k \ell} \boldsymbol{T}(k, \ell) \boldsymbol{T}(p, q)\right. \\
& +\cdots \text { multiple products } \cdots]|0\rangle
\end{aligned}
$$

where we have used the following property of the excitation operators:

$$
\boldsymbol{E}(k, \ell) \boldsymbol{E}(p, q)\left|\Phi_{I}^{P}\right\rangle=|\Phi(k+p, \ell+q)\rangle .
$$

The multiple products in equation (14) contain terms like $(1 / 6) \boldsymbol{T}_{1}^{3}$. In the following these terms will be neglected.

\section{Shifts in MR-CEPA}

The diagonal shifts in equation (4) originate from the unlinked contributions of the higher than double excitations belonging to the $\mathrm{R}$-space. The various possibilities for choosing the MR-CEPA variants may concisely be represented by introducing the shift matrices $\boldsymbol{U}^{k \ell}$, which select the correlation energy contributions to be included in the diagonal shift for $\Phi_{J}^{Q}(k, \ell)$ :

$$
K_{J}(k, \ell)=\sum_{p q} U_{p q}^{k \ell} \varepsilon(p, q)
$$

As shown in [15], in the MRCEPA0 method any combination of excitations from the $\mathrm{P}$ space to the Q space, as in equation (14), is assumed to generate an $\mathrm{R}$ space function. In equation (4) this corresponds to taking

$$
K_{J}=\sum_{k \ell} \varepsilon(k, \ell)=\boldsymbol{E}_{C} \quad \text { or } \quad U_{p q}^{k \ell}=1
$$

However, the combined excitation only belongs to the $\mathrm{R}$ space if $k+p>2$ or $\ell+q>2$. Since the interactions of the $\Phi_{I}^{P}$ with any Q-space function $\Phi(k+p, \ell+q)$ with $k+p \leqslant 2$ and $\ell+q \leqslant 2$ are already present in the MRCI equations (the first term of equation (4)), they should not contribute to the diagonal shift. These are the variationally included terms, which are included in the Q-space of the MR-CEPA wavefunction via the T-matrices, cf. equation (14). As a consequence, the shift should depend on the excitation class of $\Phi_{J}^{Q}$, thus including only those contributions not present in the Q-space, i.e. taking the $\mathbf{U}$ matrix to be complementary to the $\mathbf{T}$ matrix:

$$
K_{J}(k, \ell)=\sum_{p q}\left(1-T_{p q}^{k \ell}\right) \varepsilon(p, q) \quad \text { or } \quad U_{p q}^{k \ell}=1-T_{p q}^{k \ell} .
$$


With this choice, we have the MRDCEPA equations [15]. In the MRDCEPA no account is taken of the EPV terms. In the MR-ACPF and MR-AQCC methods the EPV terms are avoided in an average way by using a damping factor $d_{n}$, which depends on the total number of correlated electrons $n$. Following Ahlrichs [22], we gather all the mentioned methods in a table. Note, however, that in the MR-ACPF and MR-AQCC methods $E_{\mathrm{C}}$ is defined by taking the energy difference with respect to a variationally determined $\Psi_{0}$ in contrast to MRCEPA0, MRDCEPA and MR-CC, where the projection to the reference space is used.

We then have for all values of $k, \ell, p$ and $q$ :

$$
\begin{aligned}
\text { MRCI: } & U_{p q}^{k \ell}=0 \\
\text { MRCEPA0: } & U_{p q}^{k \ell}=1 \\
\text { MR-ACPF: } & U_{p q}^{k \ell}=d_{n}^{\mathrm{ACPF}}=\frac{n-2}{n} \\
\text { MR-AQCC: } & U_{p q}^{k \ell}=d_{n}^{\mathrm{AQCC}}=\frac{(n-2)(n-3)}{n(n-1)} \\
\text { MRDCEPA: } & U_{p q}^{k \ell}=1-T_{p q}^{k \ell} .
\end{aligned}
$$

In all these methods the invariance to orbital transformations within the orbital subsets is kept intact.

We will now combine the effects of the EPV terms and the VI corrections. The first method is analogous to the MR-ACPF and MR-AQCC methods, i.e. we use damping factors for the U-matrix elements analogous to the P-matrices used by Szalay et al. for the MR-AQCC-mc method [23]. This will be called the MR-ACEPA method. Our choice is almost identical to the MR-AQCC-mc one. The U-matrices to be used are given in table 1. In deriving the active orbital contributions to the EPV factor, we have used the same formulas as for the inactive orbitals. The latter ones are taken over from Szalay [23]. However, there is a difference for the combination $k=p=1$, where for both $(k, \ell)$ and $(p, q)$ one electron is excited from the inactive orbitals. Since the coincidences in the inactive and active orbital spaces respectively are independent of each other, the $U_{1 q}^{1 \ell}$ shift contribution is taken as the product of the inactive and the active orbital factors, leading to an EPV correction for this case, whereas in the MR-AQCC-mc method $U_{1 \ell^{\prime}}^{1 \ell}=1$ [23].

The second method is analogous to CEPA1 [8] in the sense that the EPV corrections are expressed in terms of individual pair energies. We call this method MR-CEPA1.

Since there is no clear-cut way to define pair energies for the active space orbitals, the CEPA1 technique can only be applied to the inactive space, i.e. to the classes $(1, \ell)$ and $(2, \ell)$, which are analogous to the single reference single and double excitations respectively. In this case the $(k=1, p=2),(k=2, p=1)$ and $(k=2$, $p=2$ ) combinations (the $A_{i}$ and $B_{i}$ blocks in table 1) are not represented by a damping factor. Instead we define the pair excitation operators $\boldsymbol{E}_{i j}$, which create two holes in the inactive orbitals $i$ and $j$. The $\boldsymbol{E}_{i j}$ generate the pair excitation functions $\left|\Phi_{J}^{Q}(i j)\right\rangle$ and the pair correlation energy contributions $\varepsilon_{i j}$ are then

\begin{tabular}{|c|c|c|c|c|c|c|c|c|c|c|}
\hline & $p$ & 0 & 0 & 0 & 1 & 1 & 1 & 2 & 2 & 2 \\
\hline & $q$ & 0 & 1 & 2 & 0 & 1 & 2 & 0 & 1 & 2 \\
\hline$k$ & $\ell$ & & & & & & & & & \\
\hline 0 & 0 & 0 & 0 & 0 & 0 & 0 & 0 & 0 & 0 & 0 \\
\hline 0 & 1 & 0 & 0 & $A_{a}$ & 0 & 0 & $B_{a}$ & 0 & 0 & 1 \\
\hline 0 & 2 & 0 & $A_{a}$ & $A_{a}$ & 0 & $B_{a}$ & $B_{a}$ & 0 & 1 & 1 \\
\hline 1 & 0 & 0 & 0 & 0 & 0 & 0 & 0 & $B_{i}$ & $B_{i}$ & $B_{i}$ \\
\hline 1 & 1 & 0 & 0 & $B_{a}$ & 0 & 0 & $C_{i} * C_{a}$ & $B_{i}$ & $B_{i}$ & $B_{i}$ \\
\hline 1 & 2 & 0 & $B_{a}$ & $B_{a}$ & 0 & $C_{i} * C_{a}$ & $C_{i} * C_{a}$ & $B_{i}$ & $B_{i}$ & $B_{i}$ \\
\hline 2 & 0 & 0 & 0 & 0 & $B_{i}$ & $B_{i}$ & $B_{i}$ & $A_{i}$ & $A_{i}$ & $A_{i}$ \\
\hline 2 & 1 & 0 & 0 & 1 & $B_{i}$ & $B_{i}$ & $B_{i}$ & $A_{i}$ & $A_{i}$ & $A_{i}$ \\
\hline 2 & 2 & 0 & 1 & 1 & $B_{i}$ & $B_{i}$ & $B_{i}$ & $A_{i}$ & $A_{i}$ & $A_{i}$ \\
\hline
\end{tabular}
obtained by:

$$
\varepsilon_{i j}=\sum_{J} c_{J}\left\langle\Psi_{0}|\boldsymbol{H}| \Phi_{J}^{Q}(i j)\right\rangle
$$

Table 1. $\quad U_{p q}^{k \ell}$ in $K_{J}(k, \ell)=\sum_{p q} U_{p q}^{k \ell} \varepsilon(p, q)$ for MR-ACEPA.

$A=\frac{(n-2)(n-3)}{n(n-1)} \quad B=\frac{n-2}{n} \quad C=\frac{n-1}{n}$

$A_{i}, B_{i}, C_{i}$ with $n_{i}=$ number of inactive electrons,

$A_{a}, B_{a}, C_{a}$ with $n_{a}=$ number of active electrons. 
Orbital correlation contributions $\varepsilon_{i}$ are defined analogously by:

$$
\varepsilon_{i}=\sum_{J} c_{J}\left\langle\Psi_{0}|\boldsymbol{H}| \Phi_{J}^{Q}(i)\right\rangle
$$

where $\Phi_{J}^{Q}(i)$ represents an excitation with one hole in orbital $i$. The summations in equations (19) and (20) include all possibilities for the number of particles since these were also all included in the shifts corresponding to the $\mathrm{A}_{i}$ and $\mathrm{B}_{i}$ blocks of table 1 . In the single reference CEPA1 method these contributions are not used, since they vanish because of the Brillouin Theorem, assuming HF orbitals are used. In the multi-reference case, however, the Generalized Brillouin Theorem may only be used for certain linear combinations of the $\left|\Phi_{J}^{Q}(i)\right\rangle[24]$.

All combinations except those corresponding to the $\mathrm{A}_{i}$ and $\mathrm{B}_{i}$ blocks in table 1 are treated in the same way as in the MR-ACEPA method, i.e. as in table 1. The shift for a 2-hole excitation $\Phi^{Q}(i j)$ is taken as [17-19, 22]

$$
\begin{aligned}
K_{i j}(2, \ell)= & \sum_{p q} U_{p q}^{2 \ell} \varepsilon(p, q)-\frac{1}{4}\left[\sum_{k=1}^{\text {ndoc }}\left(\varepsilon_{i k}+\varepsilon_{j k}\right)+\varepsilon_{i i}+\varepsilon_{j j}\right] \\
& -\frac{1}{2}\left(\varepsilon_{i}+\varepsilon_{j}\right)
\end{aligned}
$$

where the summations are over the inactive orbitals and $U_{2 q}^{2 \ell}=U_{1 q}^{2 \ell}=1$.

For the shift for a 1-hole excitation $\Phi^{Q}(i)$ we take:

$$
K_{i}(1, \ell)=\sum_{p q} U_{p q}^{1 \ell} \varepsilon(p, q)-\frac{1}{2} \sum_{k=1}^{\text {ndoc }} \varepsilon_{i k}
$$

and $U_{1 q}^{1 \ell}=1$. With these choices our MR-CEPA1 method simplifies for the single reference closed shell case to the standard CEPA1 method.

\section{Implementation}

The formalism is implemented in the Direct CI program of Saunders et al. [1] as incorporated in the GAMESSUK package [25]. This program uses an internal occupation scheme, augmented by a model external space consisting of two orbitals. All corresponding states with at most two electrons in the external space are represented by this occupation scheme. It is held in store using a packed binary format. For this purpose a total of five 64-bit words per configuration are allocated, the CONF array, two of which are for the orbital occupations, allowing a total of 62 internal orbitals. This array is used in configuration generation and selection and in calculating the internal spin coupling coefficients [26]. The same array is used in the later stages of the calculation to store various addresses and dimensions of the CI vectors associated with the occupation pattern. In this array one may store the extra information needed in the CEPA calculation. Thus an individual treatment of each configuration is possible. All external orbitals are treated on an equal footing. It is possible to distinguish between singlet and triplet coupling of these orbitals for the 2-particle states. This array also allows the storage of pair information, in case of the closed shell CEPA variants, and the specific information for the various MR-CEPA approaches.

The pair energies and the shifts are addressed in an identical fashion. In the CONF array we store the address for the shift or pair energy. For the closed shell CEPAs this would be derived from the positions of the holes leaving two positions for the singlet/triplet spincoupling. For the MRDCEPA or MR-ACEPA variants, the number of holes and particles would be used. The MR-CEPA1 requires separate information about number of holes, number of particles and position of holes, and thus needs a more involved addressing scheme. The only parts of the program that show any difference between the CEPA variants are therefore the parts devising the addressing and using the pair energies to generate the shifts. A new CEPA variant may be implemented within half a day by an experienced programmer.

The pair energies are calculated as

$$
\boldsymbol{E}_{\text {pair }}(I)=\sum_{R}^{N_{\text {ref }}} c_{R} \sum_{i}^{\text {Extn }} c_{i}^{Q}(I)\left\langle\Phi_{R}|\boldsymbol{H}| \Phi_{i}^{Q}(I)\right\rangle
$$

where the $\Phi_{i}^{Q}$ are the external configurations, which may be in the vacuum space (0 particles), the single 1-particle space or the double 2-particle space and the summation is over the external space. The $\Phi_{R}$ are the reference configuration with their coefficients, as they are in the current CI vector. Since our formalism requires intermediate normalization, the pair energies are scaled by a factor

$$
\text { scale }=\frac{1.0}{\sum_{R}^{N_{\text {ref }}} c_{R}^{2}}
$$

The pair energies are calculated by special variants of the (iajb), (ijka) and (ijkl) $\mathbf{H} * \mathbf{C}=\mathbf{Z}$ calculating routines of the direct CI program [1]. The calculation of the pair 
energies only requires the calculation of interactions involving the reference part of the vacuum space and is thus neglible in comparison with a full $\mathbf{H} * \mathbf{C}$ matrix vector product.

The shifts are added in the Davidson diagonalization $[27,28]$ part of the program. No changes are made to the originally calculated $\mathbf{C}$ and $\mathbf{Z}$-vectors or to the diagonal of the H-matrix. As the shifts change during the optimisation process, the $\mathbf{H}$-matrix elements of the Davidson matrix have to be recalculated every time. The original matrix elements are calculated as

$$
\mathbf{H}_{i j}=\mathbf{C}_{i} * \mathbf{Z}_{j} .
$$

The shifted matrix elements are

$$
\mathbf{H}_{i j}^{\prime}=\mathbf{C}_{i} * \mathbf{Z}_{j}+\mathbf{C}_{i} * \mathbf{K} * \mathbf{C}_{j}=\mathbf{H}_{i j}+\mathbf{C}_{i} * \mathbf{K} * \mathbf{C}_{j}
$$

$\mathbf{K}$ is the diagonal shift-matrix. Similarly the diagonal of the $\mathbf{H}$-matrix used in determining a new perturbation vector has to be adapted.

As there are often very few different shifts, in the case of MR-ACEPA or MRDCEPA only nine, those parts of the $\mathbf{C}_{i} * \mathbf{C}_{j}$ products are pre-calculated and stored, so that calculating a new shifted $\mathbf{H}$-matrix requires only nine multiplication-additions per matrix element. For methods like MR-ACPF or MR-AQCC only the overlap between the projections of the CI vectors onto the reference space are required. If a variance minimizer is requested, which requires $\mathbf{Z}_{i} * \mathbf{Z}_{j}$ products, also $\mathbf{C}_{i} * \mathbf{Z}_{j}$ partial inner products have to be recalculated. Thus, as the shift matrix is diagonal, the calculation of a shifted Davidson matrix is a very cheap process.

The shifts depend on the current eigenvector, which in turn depends on the shifted Davidson matrix. It is thus advantageous to define within the Davidson iterationprocess a micro iteration process:

- "calculate current best vector"

- "calculate pair energies"

- "calculate shifts and shifted Davidson matrix"

- "calculate new current best vector"

- etc.

In general this process converges sufficiently within three iterations and the time required is insignificant compared to a full $\mathbf{H C}=\mathbf{Z}$ operation. Using this scheme, the CEPA calculation generally only takes a few iterations more than a regular CI calculation. Of course, due to the linear dependence of the CEPA energy of the CI coefficients, the convergence on the CI vector has to be tightened to ensure 6 decimals accuracy in the energy, which will give rise to a few more matrix-vector products.

\section{Calculations}

The test calculations are focused on model systems for which a MR approach is necessary for a proper description. Most of these model systems have been previously adopted in the literature and we follow them closely. The results are compared with the MR-CCSD and FCI calculations, where available. The first four example calculations follow Szalay and co-workers [12]. However, we found a small discrepancy in the way the reference space had been generated and the one presented in the article. For all examples it comprises a set of symmetry non-restricted but spin adapted configuration state functions (CSFs) within a certain choice of active molecular orbitals.

Additionally, we checked the robustness of our MRCEPA variants with respect to the size consistency for open shell systems with near-degeneracy, i.e. two oxygen molecules $\left({ }^{3} \sum_{\mathrm{g}}^{-}\right)$at infinity.

\subsection{Symmetric cleavage of water molecule}

The equilibrium parameters of the water molecule (valence angle $110.565240^{\circ}$, bond length $R_{e}=$ 1.84345 a.u.) $[12,29]$ were chosen as a starting point. Subsequently, the two $\mathrm{OH}$ bonds were stretched simultaneously keeping the angle constant. All the calculations were carried out with a cc-pVDZ basis set using spherical harmonics.

For a good qualitative description a minimal $(4 \times 4)$ CAS reference space was required including the $3 \mathrm{a}_{1}, 4 \mathrm{a}_{1}$, $1 b_{2}$ and $2 b_{2}$ orbitals. The data are summarized in table 2 and illustrated in figure 1 .

MR-CEPA1 and MR-ACEPA both describe the symmetric cleavage of the molecule well. At large distances the MR-CEPA1 is closest to the multireference coupled cluster, whereas near equilibrium the average variant performs marginally better. They are very close to the MR-CC results and are also quite parallel to the FCI. Test calculations with the MRAQCC-mc method yield a curve lower than the MRCEPA1, which is thus farther from the MR-CC curve, but closer to Full CI.

\subsection{Eight-atom hydrogen cluster}

This model system was proposed to test the applicability of a method in the case of near-degeneracy. The character of the wave function can be gradually changed by varying a parameter alpha, which is responsible for the geometric definition of the $\mathrm{H}_{8}$ cluster [30]. We used Huzinaga's (5s)-[2s] [31] DZ contraction, as described in [12]. Three configurations were included in the $(2 \times 2)$ 
Table 2. Total energies in atomic units for symmetric stretching of the ground state water molecule. $R_{e}=1.84345$ a.u. is the equilibrium $\mathrm{OH}$ distance.

\begin{tabular}{lcccccc}
\hline & $1.0 R_{e}$ & $1.5 R_{e}$ & $2.0 R_{e}$ & $2.5 R_{e}$ & $3.0 R_{e}$ & $100 R_{e}$ \\
\hline MRDCI & -76.237179 & -76.068040 & -75.948222 & -75.915029 & -75.909099 & -75.907489 \\
MR-ACPF & -76.242480 & -76.073110 & -75.952000 & -75.918202 & -75.912128 & -75.910473 \\
MR-AQCC & -76.241236 & -76.071914 & -75.951117 & -75.917466 & -75.911426 & -75.909783 \\
MRDCEPA & -76.242988 & -76.073448 & -75.952571 & -75.918833 & -75.912766 & -75.911111 \\
MR-ACEPA & -76.240677 & -76.071291 & -75.950835 & -75.917305 & -75.911290 & -75.909652 \\
MR-CEPA1 & -76.240376 & -76.070980 & -75.950588 & -75.917090 & -75.911083 & -75.909449 \\
MR-CCSD & -76.240656 & -76.071345 & -75.950818 & -75.917136 & -75.911064 & - \\
FCI & -76.241860 & -76.072348 & -75.951665 & -75.917985 & -75.911946 & -75.910300 \\
\hline
\end{tabular}

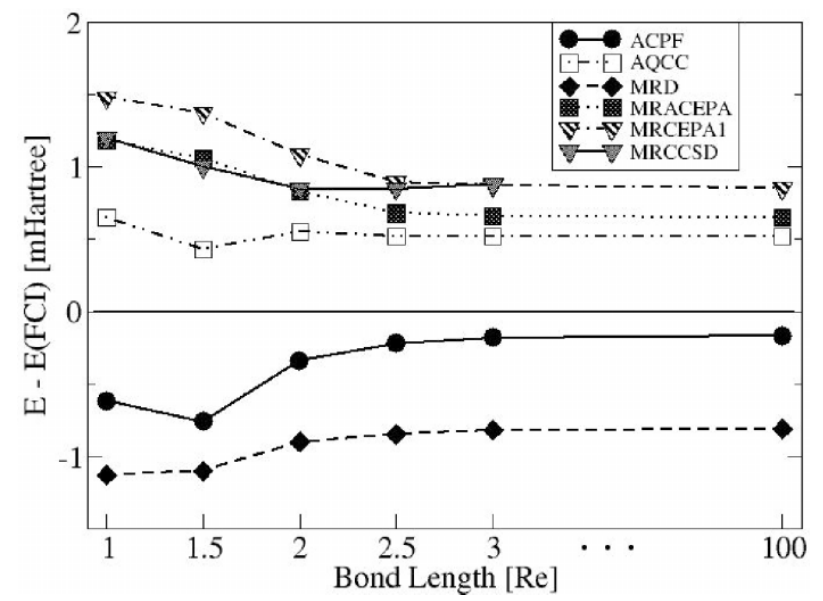

Figure 1. Potential energy surface for water molecule cleavage.

reference space, i.e.

$$
\begin{aligned}
& \left|\left(1 a_{g}\right)^{2}\left(1 b_{3 u}\right)^{2}\left(1 b_{2 u}\right)^{2}\left(2 a_{g}\right)^{2}\right| \\
& \left|\left(1 a_{g}\right)^{2}\left(1 b_{3 u}\right)^{2}\left(1 b_{2 u}\right)^{2}\left(1 b_{1 g}\right)^{2}\right| \\
& \left|\left(1 a_{g}\right)^{2}\left(1 b_{3 u}\right)^{2}\left(1 b_{2 u}\right)^{2}\left(1 b_{1 g}\right)^{1}\left(2 a_{g}\right)^{1}\right|
\end{aligned}
$$

The results in figure 2 and table 3 show that the MRDCEPA and MR-ACPF exhibit a poor performance. They overshoot the FCI result. MR-AQCC is slightly better, even though, the numbers are scattered in the neighbourhood of the FCI curve. MR-CEPA1 and MR-ACEPA follow closely the shape of the MR-CC curve. Furthermore, they do not overshoot FCI.

\subsection{The $\mathrm{Be} / \mathrm{H}_{2}$ system}

This is another typical example where a multiconfigurational approach is needed because of near-degeneracy. When the $\mathrm{Be}$ is far from the hydrogen molecule, $\left|\left(1 a_{1}\right)^{2}\left(2 a_{1}\right)^{2}\left(3 a_{1}\right)^{2}\right|$ is the dominant configuration. The

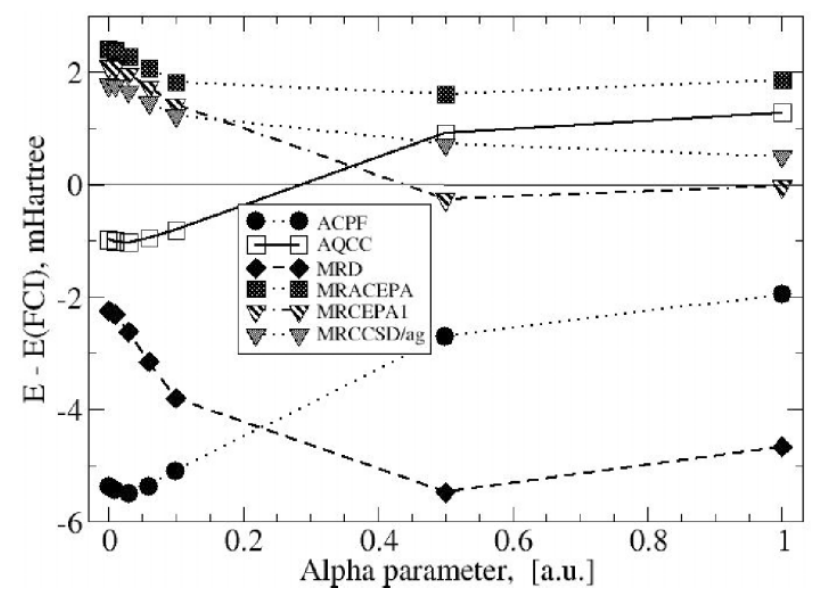

Figure 2. Potential energy surface for the $\mathrm{H}_{8}$ model system. The MR-CCSD $/ a_{\mathrm{g}}$ curve is copied from [12].

insertion of the $\mathrm{Be}$ atom into the hydrogen molecule results in a more important $\left|\left(1 \mathrm{a}_{1}\right)^{2}\left(2 \mathrm{a}_{1}\right)^{2}\left(1 \mathrm{~b}_{2}\right)^{2}\right|$ configuration. The geometrical parameters are published in [32]. An ANO basis set proposed by WidmarkMalmquist-Roos [33] was adopted as valence triple zeta plus double polarization contraction $(\mathrm{H}: 3 \mathrm{~s} 2 \mathrm{p}$, Be: $4 \mathrm{~s} 3 \mathrm{p} 2 \mathrm{~d})$ using spherical harmonics. A $(2 \times 2)$ CASSCF calculation was carried out and the same active orbitals were used to generate the reference space $\left(\left|\ldots 3 a_{1}^{2} 1 b_{2}^{0}\right|\right.$, $\left|\ldots 3 a_{1}^{0} 1 b_{2}^{2}\right|$ and $\left.\left|\ldots 3 a_{1}^{1} 1 b_{2}^{1}\right|\right)$ in all subsequent $M R$ calculations. The FCI total energies were also obtained. The results are listed in figure 3 and table 4 .

\subsection{Singlet-triplet separation of methylene $\left(\mathrm{CH}_{2}\right)$}

We used the FCI results obtained by Bauschlicher and Taylor [34] as a benchmark of the energy difference between the ${ }^{1} \mathrm{~A}_{1}$ and ${ }^{3} \mathrm{~B}_{1}$ states of $\mathrm{CH}_{2}$. The DZP basis set was exploited with core orbitals frozen at CASSCF level. Two different reference spaces were considered: one generated with and another without symmetry restriction, for the set of $3 a_{1}$ and $1 b_{1}$ orbitals occupied 
Table 3. Dependence of the total ground state energy of $\mathrm{H}_{8}$ model system of the parameter $\alpha$ in Hartrees.

\begin{tabular}{lcccccccc}
\hline$\alpha$ & MRDCI & MR-ACPF & MR-AQCC & MRDCEPA & MR-ACEPA & MR-CEPA1 & $*$ MR-CCSD/a & FCI \\
\hline 0.0001 & -4.300005 & -4.313412 & -4.309024 & -4.310277 & -4.305637 & -4.305922 & -4.306265 & -4.308040 \\
0.001 & -4.300025 & -4.313441 & -4.309050 & -4.310303 & -4.305661 & -4.305946 & -4.306289 & -4.308064 \\
0.003 & -4.300075 & -4.313509 & -4.309111 & -4.310370 & -4.305719 & -4.306005 & -4.306347 & -4.308122 \\
0.006 & -4.300156 & -4.313627 & -4.309215 & -4.310490 & -4.305819 & -4.306107 & -4.306445 & -4.308218 \\
0.01 & -4.300283 & -4.313800 & -4.309371 & -4.310679 & -4.305976 & -4.306265 & -4.306598 & -4.308362 \\
0.03 & -4.301244 & -4.314853 & -4.310383 & -4.311977 & -4.307079 & -4.307382 & -4.307692 & -4.309354 \\
0.06 & -4.303579 & -4.316999 & -4.312587 & -4.314779 & -4.309561 & -4.309900 & -4.310161 & -4.311628 \\
0.1 & -4.307870 & -4.320912 & -4.316627 & -4.319616 & -4.313996 & -4.314396 & -4.314577 & -4.315812 \\
0.5 & -4.361839 & -4.372788 & -4.369172 & -4.375571 & -4.368477 & -4.370349 & -4.369373 & -4.370090 \\
1 & -4.408469 & -4.418498 & -4.415255 & -4.421200 & -4.414673 & -4.416572 & -4.416033 & -4.416537 \\
\hline
\end{tabular}

*The numbers for MRCCSD/a $/ \mathrm{a}_{\mathrm{g}}$ are copied from [12].

Table 4. FCI relative energies in $\mathrm{mHartree}$ for the ground state of $\mathrm{Be} / \mathrm{H}_{2}$ system. Inter-atomic distances in Bohrs. The values of the FCI energies in Hartree are shown in the last row.

\begin{tabular}{lccc}
\hline $\mathrm{R}\left(\mathrm{BeH}_{2}\right)$ & 2.50 & 2.75 & 3.00 \\
$\mathrm{R}\left(\mathrm{H}_{2}\right)$ & 2.78 & 2.55 & 2.32 \\
$\mathrm{MRCI}$ & 2.900 & 3.503 & 4.609 \\
$\mathrm{MR}-A C P F$ & -1.402 & -1.773 & -1.081 \\
$\mathrm{MR}-A Q C C$ & 0.465 & 0.576 & 1.494 \\
$\mathrm{MRDCEPA}$ & -2.9764 & -3.2721 & -4.5424 \\
$\mathrm{MR}-A C E P A$ & 1.0376 & 1.2178 & 1.1196 \\
$\mathrm{MR}-\mathrm{CEPA} 1$ & 1.6890 & 2.1467 & 2.0485 \\
$\mathrm{MR}-\mathrm{CCSD} / \mathrm{a}_{1} *$ & 1.6750 & 2.2490 & 1.7390 \\
$\mathrm{MR}-\mathrm{CCSD} / \mathrm{b}_{2}{ }^{*}$ & 1.2680 & 1.8900 & 2.4690 \\
$\mathrm{FCI}$ & -15.698037 & -15.671616 & -15.678210 \\
\hline
\end{tabular}

*MRCCSD $/ a_{1}:\left|\ldots 3 a_{1}^{2} 1 b_{2}^{0}\right|$ as vacuum state; MRCCSD $/ b_{1}:\left|\ldots 3 a_{1}^{0} 1 b_{2}^{2}\right|$ as vacuum state.



Figure 3. Energies in mHartree for the ground state $\mathrm{Be} / \mathrm{H}_{2}$ system, cf. table 4 .

with two electrons. In the symmetry restricted case, we have only one configuration for the triplet $\left(3 a_{1}^{1} 1 b_{1}^{1}\right)$ and two for the singlet $\left(3 a_{1}^{2} 1 b_{1}^{0}, 3 a_{1}^{0} 1 b_{1}^{2}\right)$ state. In the symmetry non-restricted case, the reference space comprises three different CSFs $\left(3 a_{1}^{2} 1 b_{1}^{0}, 3 a_{1}^{0} 1 b_{1}^{2}\right.$ and $\left.3 a_{1}^{1} 1 b_{1}^{1}\right)$. All results are compiled in table 5 .

\section{5. $\mathrm{Two} \mathrm{O}_{2}$ molecules}

As a particularly challenging example, the size consistency of various methods was tested using two $\mathrm{O}_{2}\left({ }^{3} \Sigma_{\mathrm{g}}^{-}\right)$ molecules $\left(R_{e}=1.2 \AA\right)$ at a large distance from each other. Because of the near-degeneracy in $\mathrm{O}_{2}\left({ }^{3} \Sigma_{\mathrm{g}}^{-}\right)$the deviations from size consistency may be expected to be relatively large. The calculations were carried out with the standard DZP basis set. The monomer reference function was generated by performing a CASSCF calculation with six electrons in the $1 \pi_{\mathrm{xu}}, 1 \pi_{\mathrm{yu}}, 1 \pi_{\mathrm{xg}}$ and $1 \pi_{\mathrm{yg}}$ MOs. The $1 \sigma_{\mathrm{g}}$ and $1 \sigma_{\mathrm{u}}$ MOs were frozen to the RHF level. For the dimer calculations the two-monomer CASSCF functions were combined into a quintet function. All calculations were carried out without imposing symmetry restrictions on the reference set used for generating the excitations. The results are given in table 6 .

From table 6 it appears that using either the Davidson or the Pople corrections cannot compensate for the large errors in the MRCI calculations. The MR-ACPF and MR-AQCC methods also lead to large errors. All methods including the VI corrections, however, lead to much smaller errors $(<1 \mathrm{mH})$, which are all of the same order of magnitude. 
Table 5. Total energies in Hartree for the singlet ${ }^{1} \mathrm{~A}_{1}$ and triplet ${ }^{3} \mathrm{~B}_{1}$ state of methylene for the two CAS choices. The excitation energies $\Delta E$, i.e. the energy difference between ${ }^{1} \mathrm{~A}_{1}$ and ${ }^{3} \mathrm{~B}_{1}$ states, are translated into $\mathrm{kcal} / \mathrm{mol}$.

\begin{tabular}{|c|c|c|c|c|c|c|}
\hline & \multicolumn{2}{|c|}{${ }^{1} \mathrm{~A}_{1}$} & \multicolumn{2}{|c|}{${ }^{3} \mathrm{~B}_{1}$} & \multicolumn{2}{|c|}{$\mathrm{E}, \mathrm{kcal} / \mathrm{mol}$} \\
\hline & Sym CAS & Nonsym CAS & Sym CAS & Nonsym CAS & Sym CAS & Nonsym CAS \\
\hline MRCI & -39.022156 & $-39.022357^{*}$ & -39.041602 & -39.041699 & 12.20 & 12.14 \\
\hline MR-ACPF & -39.026176 & -39.026416 & -39.045425 & -39.045538 & 12.08 & 12.00 \\
\hline MR-AQCC & -39.024493 & -39.024715 & -39.043823 & -39.043929 & 12.13 & 12.06 \\
\hline MRDCEPA & -39.028375 & -39.028636 & -39.047385 & -39.047506 & 11.93 & 11.84 \\
\hline MR-ACEPA & -39.024519 & -39.024739 & -39.043726 & -39.043832 & 12.05 & 11.98 \\
\hline MR-CEPA1 & -39.025238 & -39.025465 & -39.044214 & -39.044322 & 11.91 & 11.83 \\
\hline SSMRCC & \multicolumn{2}{|c|}{$-39.044064 * *$} & \multicolumn{2}{|c|}{-39.025400} & \multicolumn{2}{|c|}{12.00} \\
\hline FCI & \multicolumn{2}{|c|}{-39.027183} & \multicolumn{2}{|c|}{-39.046260} & \multicolumn{2}{|c|}{11.97} \\
\hline
\end{tabular}

*The numbers in bold reproduce the data from [12].

**Result from [12]. The low-spin reference function as Fermi vacuum for the triplet state $\left({ }^{3} \mathrm{~B}_{1}\right)$ and the amplitude of its low-spin pair forced to be 1.0 .

***Sym CAS implies that the CAS reference function was symmetry adapted, before generating the SDCI space; Nonsym implies that all configurations in the CAS space are used in the configuration generation without regard to symmetry.

Table 6. Energies and size consistency errors (in $\mathrm{mH}$ ) for $\left(\mathrm{O}_{2}\right)_{2}$. No symmetry restrictions are applied in generating the CAS reference space.

\begin{tabular}{lcrr}
\hline & Monomer & Dimer & SC-error \\
\hline MRCI & -149.973622 & -299.909624 & 37.62 \\
Davidson & -150.001007 & -299.993109 & 8.91 \\
Pople & -150.000490 & -300.014397 & 13.42 \\
MR-ACPF & -149.998138 & -300.006900 & -10.62 \\
MR-AQCC & -149.992983 & -299.995068 & -9.10 \\
MRDCEPA & -149.999653 & -300.000132 & -0.83 \\
MR-ACEPA & -149.990355 & -299.981093 & -0.38 \\
MR-CEPA1 & -149.990391 & -299.981279 & -0.50 \\
\hline
\end{tabular}

We therefore conclude that the inclusion of the VI corrections is essential for obtaining a method, which is nearly size-consistent. The performance of the MR-CEPA1 method is not really better than that of the MR-ACEPA method, even though the EPV corrections are handled in a formally better way.

\section{Conclusions}

We combined the previously suggested corrections for 'variationally included' and 'exclusion principle violating' (EPV) terms to arrive at two new MR-CEPA variants. The corrections for the VI terms, as already implemented in the MRDCEPA, alleviate the bulk of the size-inconsistency of the MRCI. The EPV terms then damp the corrections to get closer to the MR-CC results. The resulting MR-CEPA1 and MRACEPA yield comparable results that are consistently close to the MR-CC. As the MR-ACEPA, which is akin to Szalay's MR-AQCC-mc approach, is the most simple to implement, it is the preferred MR-CEPA method.

No MR-CEPA method is exactly size-consistent; we believe that the MR-ACEPA method is the best MR-CEPA method that one can realistically attain using only diagonal shifts.

\section{Acknowledgements}

We thank P. Szalay for stimulating and helpful discussions and correspondence.

\section{References}

[1] V.R. Saunders, J.H. van Lenthe. Mol. Phys., 48, 923 (1983).

[2] P.E.M. Siegbahn. J. chem. Phys., 72, 1647 (1980).

[3] P.E.M. Siegbahn. Int. J. quantum Chem., 18, 1229 (1980).

[4] W. Duch, J. Karwowski. Comput. Phys. Rep., 2, 93 (1985). 
[5] H. Lischka, H. Dachsel, R. Shepard, R. Harrison. The Parallelization of a General Ab Initio Multireference Configuration Interaction Program - the COLUMBUS Program System, American Chemical Society, Washington, DC (1995).

[6] J. Cizek, J. Paldus, L. Sroubková. Int. J. quantum Chem., 3, 149 (1969).

[7] A.C. Hurley. Electron Correlation in Small Molecules, Academic Press, New York (1976).

[8] R. Ahlrichs, P. Scharf. In The Coupled Pair Approximation: Ab Initio Methods in Quantum Chemistry, K.P. Lawley (Ed.), Vol. 1, Wiley \& Sons, New York (1987).

[9] J. Paldus, L. Pylypow, B. Jeziorski. In Many Body Methods in Quantum Chemistry, Lecture Notes in Chemistry, U. Kaldor (Ed.), Vol. 52, Springer, Heidelberg (1989).

[10] P. Piecuch, X. Li, J. Paldus. Chem. Phys. Lett., 224, 267 (1994).

[11] P. Piecuch, N. Oliphant, L. Adamowicz. J. chem. Phys., 99, 1875 (1993).

[12] M. Kallay, P.G. Szalay, P.R. Surján. J. chem. Phys., 117, 980 (2002).

[13] R. Gdanitz, R. Ahlrichs. Chem. Phys. Lett., 143, 413 (1988).

[14] P.G. Szalay, R.J. Bartlett. Chem. Phys. Lett., 214, 481 (1993).

[15] P.J.A. Ruttink, J.H. van Lenthe, R. Zwaans, G.C. Groenenboom. J. chem. Phys., 94, 7212 (1991).

[16] W.D. Laidig, R.J. Bartlett. Chem. Phys. Lett., 104, 424 (1984).

[17] H.P. Kelley, A.M. Sessler. Phys. Rev., 132, 2091 (1963).

[18] H.P. Kelley. Phys. Rev., 134, A1450 (1964).

[19] P.G. Szalay, R.J. Bartlett. J. chem. Phys., 103, 3600 (1995).
[20] L. Füsti-Molnar, P.G. Szalay. J. chem. Phys., 100, 6288 (1996).

[21] P.J.A. Ruttink. Private communication (2004).

[22] C. Zirz, R. Ahlrichs. Recent Developments in Coupled Pair Theories 1-83, Science Research Council UK, Daresbury (1979).

[23] L. Fusti-Molnar, P.G. Szalay. J. phys. Chem., 100, 6288 (1996).

[24] B. Levy, G. Berthier. Int. J. quantum Chem., 2, 307 (1968).

[25] M.F. Guest, J.H. van Lenthe, J. Kendrick, K. Schöffel, P. Sherwood, R.J. Harrison. GAMESS-UK, a package of Ab Initio programs (2002). With contributions from R.D. Amos, R.J. Buenker, M. Dupuis, N.C. Handy, I.H. Hillier, P.J. Knowles, V. Bonacic-Koutecky, W. von Niessen, V.R. Saunders and A.J. Stone. It is derived from the original GAMESS code due to M. Dupuis, D. Spangler and J. Wendolowski, NRCC Software Catalog, Vol. 1, Program No. QG01 (GAMESS) 1980.

[26] J. Paldus, M.J. Boyle. Phys. Scr., 21, 295 (1980).

[27] E.R. Davidson. NATO ASI Ser., Ser. C, 113, 95 (1983).

[28] E.R. Davidson. J. comput. Phys., 17, 87 (1975).

[29] J. Olsen, P. Jorgensen, H. Koch, A. Balkova, R.J. Bartlett. J. chem. Phys., 104, 8007 (1996).

[30] K. Jankowski, L. Meissner, J. Wasilwski. Int. J. quantum Chem., 28, 931 (1985).

[31] S. Huzinaga. J. chem. Phys., 42, 1293 (1965).

[32] G.D. Purvis, R. Shepard, F.B. Brown, R.J. Bartlett. Int. J. quantum Chem., 23, 835 (1983).

[33] P.-O. Widmark, P.-A. Malmquist, B.O. Roos. Theor. Chim. Acta, 79, 419 (1991).

[34] J.Ch.W. Bauschlicher, P.R. Taylor. J. chem. Phys., 85, 5410 (1986). 